\title{
Behavioural problems in infants and toddlers were associated with later psychiatric disorders
}

\author{
Keenan K, Shaw D, Delliquadri E, et al. Evidence for the continuity of early problem behaviors: application of a developmental \\ model.J Abnorm Child Psych 1998 Dec;26:441-54.
}

\section{Question}

Do problem behaviours at 1-3 years of age predict psychopathology at 5 years of age?

\section{Design}

Inception cohort followed for up to 4 years.

\section{Setting}

Allegheny County, Pennsylvania, USA.

\section{Patients}

104 mothers and children (58\% boys, age range $6-11$ mo, $61 \%$ white) who were recruited from a federally funded nutritional programme for low income families with young children. Children with severe handicaps or known sensori-motor deficits were excluded. Follow up at 5 years of age was $86 \%$.

\section{Assessment of prognostic factors}

Difficult temperament, child non-compliance, and aggression were assessed in a laboratory at 12, 18, and 24 months of age. Child temperament was assessed using an abbreviated Infant Characteristics Questionnaire, and aggression and noncompliance with assessments of mother-child interaction. The Child Behavior Checklist (CBCL) was completed at 36 months of age by mothers. Assessors were blind to the study hypotheses.

\section{Main outcome measures}

Symptoms related to DSM-III-R internalising and externalising disorders were assessed by using the Kiddie-Schedule for Affective Disorders when the children were 5 years of age.

\section{Main results}

At 5 years of age, $26 \%$ of children met criteria for $\geqslant 1$ DSM-III-R disorder (15\% externalising disorders, $15 \%$ internalising disorders) and an additional $30 \%$ of children had subthreshold disorders $(>50 \%$ but $<100 \%$ of required symptoms) $(15 \%$ externalising, 24\% internalising). All except 4 of 30 statistically significant correlations supported continuity among early problem behaviours and differentiation among externalising and internalising problems and their precursors. In girls at 5 years of age, internalising disorders were associated with difficult temperament at 18 months $(r=0.34, \mathrm{p}<0.05)$, and externalising disorders were associated with CBCL externalising score at 36 months $(r=0.41, \mathrm{p}<0.05)$. In boys who were 5 years of age, internalising disorders were positively associated with difficult temperament at 24 months $(r=0.28, \mathrm{p}<0.05)$ and CBCL internalising score at 36 months $(r=0.26, \mathrm{p}<0.05)$ and were negatively associated with non-compliance at 24 months $(r=-0.25, \mathrm{p}<0.05)$. Externalising disorders in boys were positively associated with aggression at 18 months $(r=0.30$, $\mathrm{p}<0.05)$ and CBCL externalising score at 36 months $(0.28$, $\mathrm{p}<0.05)$ and were negatively associated with difficult temperament at 18 months $(r=-0.28, \mathrm{p}<0.05)$.

\section{Conclusion}

Difficult temperament, non-compliance, and aggression in infancy and toddlerhood (ages 1-3 y) were associated with internalising and externalising psychiatric disorders at 5 years of age among children from low income families.

Source of funding: in part, National Institute of Mental Health.

For correspondence:Dr K Keenan, Department of Psychiatry-MC3077, University of Chicago, 5841 South Maryland Avenue, Chicago, IL 60637, USA. Fax +1 7737029929.

\section{Commentary}

This study by Keenan et al focuses on the continuity of early problem behaviours, an issue of importance to professionals working in the area of children's mental health. The authors begin with a sensible developmental model that accounts for the shifting presentation of difficult behaviours over time and then test specific predictions based on that model.

The main conclusions of this study are that evidence exists for continuity of early emotional and behavioural problems and support for early differentiation between internalising and externalising problems. Difficult temperament is associated with later internalising problems and aggression and non-compliance with later externalising problems.

The authors acknowledge the study's limitations, which include small sample size, preferential assessment of precursors of externalising compared with internalising problems, and strong reliance on maternal reports. One major aspect of the work, however, deserves comment. The exact nature of this high risk sample is not clear. Was this a clinical subsample (attending a mental health clinic) of a high risk, non-clinic sample from the nutrition supplement programme? It is only with clarity in sample definition that replication can be undertaken and appropriate generalisability of results communicated.

Clinicians involved in the care of young children should be aware of and actively inquire about early signs of noncompliance, aggression, and other evidence of temperamental difficulties. Assisting the parents of these children with handling these behaviours may be helpful. Options include discussing developmentally appropriate activities for parents and children to do together, encouraging appropriate limit setting and other parenting skills, and suggesting parents ensure they take some time for themselves to decrease stress levels and maintain their own wellbeing. Attention to the presence of parental health difficulties that may impact on child behaviour, such as depression, is important.

As clinicians, we need to understand (and communicate) what is known about the prognosis of early difficult behaviours among infants and preschoolers. We also need to focus on early prevention/ intervention activities to decrease later psychopathology. This study provides a step towards undertaking these tasks.

Ellen L Lipman, MD McMaster University Hamilton, Ontario, Canada 